Specificity of ENC2015 was established with co-infusion of the Factor XIIIa inhibitor, iodoacetamide. 18F-ENC2015 biodistribution, kinetics, radiometabolism and ability to bind to an acutely thrombosed artery in vivo were characterised in rats.

Results Both Cy5 and fluorine-18 labelled ENC2015 rapidly and specifically bound to both low and high shear thrombi. Both thrombus fluorescence and PET radioactivity was inhibited by a factor XIIIa inhibitor. There was no metabolism of 18F-ENC2015 for over 8 hours when incubated ex vivo in whole human blood. In vivo, approximately $42.0 \%$ of parent radiotracer remained in the blood $60 \mathrm{~min}$ post-administration. Biodistribution studies demonstrated low uptake and rapid clearance from tissues with elimination via the urinary system. In an in vivo rat model of arterial thrombosis, 18F-ENC2015 uptake was markedly increased in the thrombosed carotid artery compared to the contralateral patent artery (mean standard uptake value ratio of 2.40 versus $0.74, \mathrm{p}<0.0001$ ).

Conclusion ENC2015 rapidly and selectively binds to acute thrombus in both an ex vivo human translational model and an in vivo rodent model of arterial thrombosis. This probe holds promise for the non-invasive identification of thrombus formation in cardiovascular disease.

Figure 1. Biodistribution and thrombus binding of $18 \mathrm{~F}-$ ENC2015 in rats. Panel A shows images of the time averaged uptake within the thrombus and skin incision compared with the contralateral vessel. Panel B is a decay corrected time activity curve showing 18F-ENC2015 kinetics (mean SUV, $\mathrm{n}=3$ ) within our in vivo thrombosis model. Note the rapid blood pool clearance of 18F-ENC2015 with early uptake in the skin incision which tails off to equilibrate with the arterial thrombus between 60 and 90 mins. Panel C demonstrates the mean change in SUV ratio of the thrombosed vessel compared with contralateral vessel and LV blood pool over time $(n=3)$. Thrombus vs contralateral vessel SUV from 35 to 180 minutes $\mathrm{p}$ value $<0.0001$ (paired t-test).

Conflict of Interest None

\section{AN ANALYSIS OF EXTRA CARDIAC FINDINGS GENERATED BY THE EXPANSION OF CT CORONARY ANGIOGRAPHY AT A DISTRICT GENERAL HOSPITAL}

${ }^{1}$ Jonathan Hudson*, ${ }^{2}$ Saad Fyyaz, ${ }^{3}$ Sarojini David, ${ }^{4}$ Olaolu Olabintan, ${ }^{5}$ Athanasios Katsigris, ${ }^{6}$ Khaled Alfakih. ${ }^{1}$ University Hospital Lewisham; ${ }^{2}$ Lewisham \& Greenwich NHS Trust

10.1136/heartjnl-2019-BCS.8

Introduction The updated 2016 NICE guidelines for stable chest pain recommend CT coronary angiography (CTCA) as the first line investigation for all patients presenting with stable chest pain. The implementation of these guidelines has led to the expansion of CTCA services. The CT scanning field of view extends beyond the heart resulting in extra-cardiac findings, which can require further investigation. Our aim was to assess the impact of the expansion of CTCA on the volume of extra cardiac findings, and to quantify the frequency and cost of the downstream tests generated.

Methods We undertook a retrospective audit of all CTCAs performed between January 2017 and June 2018 at a district general hospital. Extra-cardiac findings in the CTCA report were classified according to whether they were clinically significant or insignificant. We analysed which scans required downstream investigation and follow up, and the financial implications using standardized NHS England tariffs.

Results A total of 652 scans were performed with a total of $202(26.2 \%)$ individual incidental extra-cardiac findings, 106 (16.3\%) were deemed to be clinically significant requiring further investigation or referral to a specialist clinic. The extracardiac findings were abdominal (45), thoracic (42), cardiac (14), and involving breast tissue (5). The most common specific extra-cardiac findings were liver cysts (36) and pulmonary nodules (22). Two lung malignancies was identified.

There were a total of 122 further investigations or referrals generated by extra-cardiac findings. The most common downstream investigations required were abdominal ultrasound (40), CT thorax (21), and echocardiogram (12). Referral to a specialist clinic for further assessment was required in 31 patients including 5 to a triple assessment breast clinic.

The total additional cost of imaging investigations or onwards referral to a specialist clinic was $£ 12,464$. Broken down by category, thoracic findings required $£ 7,828$, abdominal findings $£ 1,710$, cardiac findings $£ 2,226$, and breast findings $£ 700$. The added cost on average per patient for investigating and referring on extra-cardiac findings was $£ 19$.

Conclusion Our data suggests that CTCA generates a significant number of clinically relevant extra-cardiac findings that require further investigation. These have additional costs and generate significant workload for the rapid access chest pain clinic. Importantly, we identified significant delays in the downstream investigations in the absence of robust referral pathways. The expansion of CTCA to even older patients will increase the extra-cardiac findings and will require hospitals to develop referral pathways and to clearly delineate responsibility for further investigation, follow up and onwards referral of important findings. Although somewhat controversial due to ethical considerations of missing potentially pre-malignant and malignant conditions, limiting the scanning field of view to scan just the heart, may reduce the number of incidental findings and limit radiation exposure ${ }^{1}$.

\section{REFERENCE}

1. Robertson J, Nicholls S, Bardin P, et al. Incidental Pulmonary Nodules Are Common on CT Coronary Angiogram and Have a Significant Cost Impact. Heart Lung Circ 2017.

Conflict of Interest None

\section{EFFECT OF COFFEE CONSUMPTION ON ARTERIAL STIFFNESS FROM UK BIOBANK IMAGING STUDY}

${ }^{1}$ Kenneth Fung*, ${ }^{2}$ Luca Biasiolli, ${ }^{2}$ Evan Hann, ${ }^{1}$ Nay Aung, ${ }^{1}$ Jose Paiva, ${ }^{2}$ Elena Lukaschuk, ${ }^{1}$ Mihir Sanghvi, ${ }^{2}$ Valentina Carapella, ${ }^{2}$ Jennifer Rayner, ${ }^{2}$ Konrad Werys, ${ }^{2}$ Henrike Puchta, ${ }^{2}$ Katharine Thomas, ${ }^{2}$ Niall Moon, ${ }^{1}$ Mohammed Khanji, ${ }^{3}$ Stefan Neubauer, ${ }^{2}$ Stefan Piechnik,

${ }^{4}$ Patricia B Munroe, ${ }^{1}$ Steffen Petersen. 'William Harvey Research Institute, Queen Mary University of London; ${ }^{2}$ Division of Cardiovascular Medicine, University of Oxford; ${ }^{3}$ Oxford University; ${ }^{16}$ Division of Cardiovascular Medicine, University of Oxford; ${ }^{4}$ Queen Mary University of London

\subsection{6/heartjnl-2019-BCS.9}

Introduction Coffee is widely reported to be the world's most popular drink and previous studies revealed acute increases in arterial stiffness with its consumption. But, the reported 


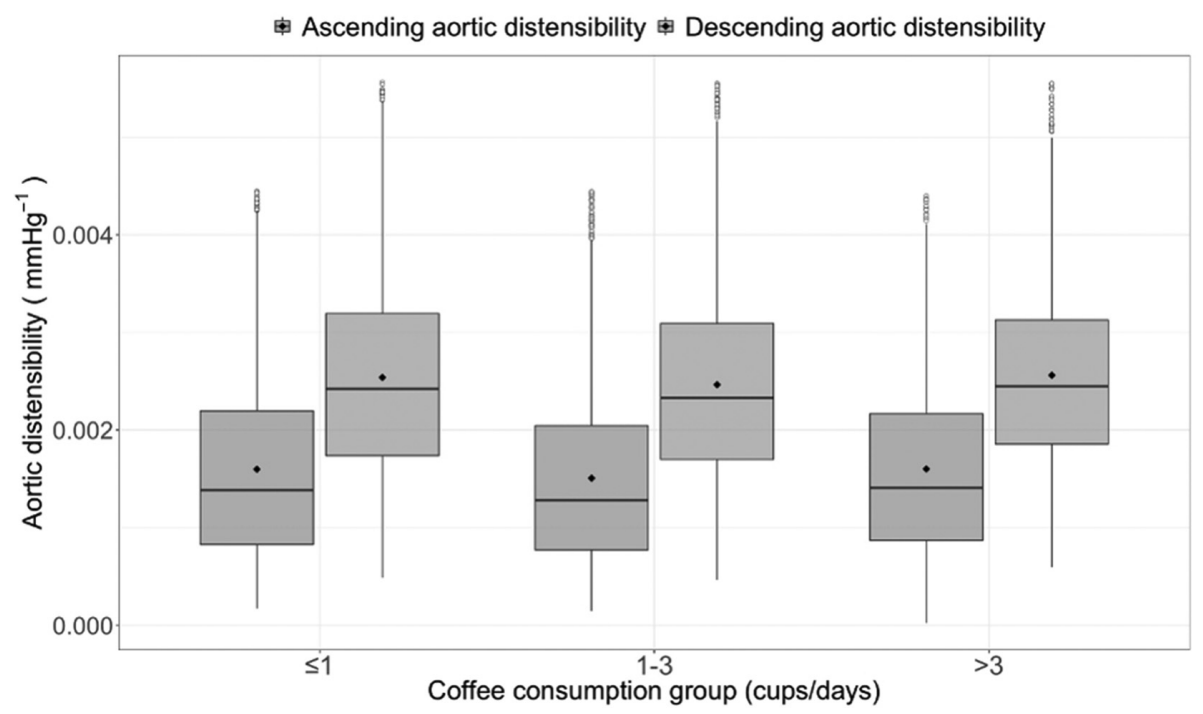

\section{Abstract 9 Figure 1}

Abstract 9 Table 1 Baseline characteristics

\begin{tabular}{|l|l|l|l|l|}
\hline & $\begin{array}{l}\text { (1 cup /day } \\
(\mathbf{n = 3 8 9 2 )}\end{array}$ & $\begin{array}{l}\mathbf{1 - 3} \text { cups/day } \\
(\mathbf{n = 2 9 7 8 )}\end{array}$ & $\begin{array}{l}\mathbf{>} \text { cups/day } \\
(\mathbf{n}=\mathbf{1 5 4 2})\end{array}$ & P value \\
\hline Age (years) & $61(7.2)$ & $62(7.3)$ & $61(7.3)$ & $<0.001$ \\
\hline Sex (male) & $1797(46.2 \%)$ & $1562(52.5 \%)$ & $873(56.6 \%)$ & $<0.001$ \\
\hline Body mass index $\left(\mathrm{kg} / \mathrm{m}^{2}\right)$ & $26(4.1)$ & $26(4.0)$ & $27(4.2)$ & $<0.001$ \\
\hline Regular alcohol intake & $1571(40.4 \%)$ & $1561(52.4 \%)$ & $777(50.4 \%)$ & $<0.001$ \\
\hline Current smoker & $106(2.7 \%)$ & $100(3.4 \%)$ & $87(5.6 \%)$ & $<0.001$ \\
\hline Systolic blood pressure $(\mathrm{mmHg})$ & $133(16.3)$ & $136(16.9)$ & $135(19.0)$ & 0.617 \\
\hline Heart rate (bpm) & $72(11.0)$ & $72(11.0)$ & $72(10.8)$ & 0.963 \\
\hline Vegetable intake (heaped tbsp/day) & $5(3.5)$ & $5(3.1)$ & $5(3.6)$ & 0.842 \\
\hline Arterial stiffness index $(\mathrm{m} / \mathrm{s})$ & $9.4(2.5)$ & $9.5(2.5)$ & $9.5(2.4)$ & 0.138 \\
\hline
\end{tabular}

Continuous variables displayed as mean (standard deviation) and categorical data are presented as numbers of participants (percentages) in each group of coffee consumption. $P$-value calculated using one-way ANOVA or Chi-square test.

Abstract 9 Table 2 Effect estimate of daily coffee consumption (compared to individuals who drink $\leq 1$ cup of coffee) on arterial stiffness after adjustments for confounders

\begin{tabular}{|l|l|l|l|l|}
\hline & \multicolumn{2}{l|}{ 1-3 cups/day } & \multicolumn{2}{l|}{$>\mathbf{3}$ cups/day } \\
\hline Arterial stiffness measure & Effect estimate* & $P$ value & Effect estimate* & $P$ value \\
\hline Ascending aortic distensibility & $-0.77 \%$ & 0.534 & $-0.73 \%$ & 0.650 \\
\hline Descending aortic distensibility & $+0.10 \%$ & 0.906 & $+0.21 \%$ & 0.844 \\
\hline Arterial stiffness index & $-0.048 \mathrm{~m} / \mathrm{s}$ & 0.417 & $-0.083 \mathrm{~m} / \mathrm{s}$ & 0.280 \\
\hline
\end{tabular}

*for AoD, effect estimates represent percentage change but for ASI, effect estimates represent absolute change in $\mathrm{m} / \mathrm{s}$

chronic effects of coffee on arterial stiffness are inconsistent and limited by modest number of studied subjects.

This study aims to evaluate the association of coffee consumption on arterial stiffness using two forms of stiffness measures in a large population cohort. Aortic distensibility (AoD) is a local measure of arterial stiffness whilst arterial stiffness index (ASI) is a measure of wave reflection. Both measures have been shown to be predictors of cardiovascular events.

Methods This cross-sectional cohort analysis comprised of 17,932 participants in the UK Biobank Imaging Study who underwent both cardiovascular magnetic resonance (CMR) imaging and pulse waveform measurements via finger probes. Participants with known cardiovascular disease were excluded. 
Coffee consumption habits were self-reported at the time of imaging and those who drink $>25$ cups/day were excluded. Coffee consumption was categorised into 3 groups $(\leq 1,1-3$, $>3$ cups/day) with the lowest group used as the reference in the analyses.

AoD was derived using an automated method to obtain the maximum and minimum luminal areas in the ascending (AA) and descending aorta (DA) from cine CMR images. ASI was calculated by dividing the participant's height by the time interval between the peaks of the waveform recorded. AoD and ASI outliers (1.5x inter-quartile range rule) were excluded. Log transformation of AoD values was performed prior to regression analyses.

Associations between coffee consumption and stiffness measures were assessed separately using univariate linear regression models adjusting for age, sex, ethnicity, Townsend deprivation index, current smoking, higher levels of education, height, weight, regular alcohol consumption (3 times/week), systolic blood pressure, resting heart rate, presence of hypertension, hypercholesterolaemia or diabetes, intake of vegetable, meat, water and tea consumption.

Results Baseline characteristics of the 8,412 participants included in the final analyses are summarised in table 1 . Moderate and heavy coffee drinkers were more likely to be male, smoke and consumed alcohol regularly. No significant differences were observed in the systolic blood pressures and heart rates between the groups.

The unadjusted distributions of AoD in AA and DA were similar across the 3 groups (figure 1). Our regression models found no statistically significant differences in all three arterial stiffness measures for individuals who drink 1-3 cups or $>3$ cups of coffee/day compared with the reference group (table 2).

Conclusion In this large middle-aged cohort without cardiovascular disease, moderate to heavy coffee consumption was not associated with significant changes in arterial stiffness measured by AoD and ASI compared with individuals who drink $\leq 1$ cups of coffee/day.

Conflict of Interest None

\section{A SIMPLE METHOD TO IMPROVE THE RELIABILITY OF ECHOCARDIOGRAPHY IN PATIENTS WITH ATRIAL FIBRILLATION}

${ }^{1}$ Karina Bunting*, ${ }^{2}$ Michael Griffith, ${ }^{3}$ Jonathan Townend, ' Gregory Lip, ${ }^{1}$ Paulus Kirchhof, ${ }^{3}$ Rick Steeds, ${ }^{1}$ Dipak Kotecha. ' University of Birmingham; ${ }^{2}$ Queen Elizabeth Hospital Birmingham; ${ }^{3}$ University Hospital Birmingham

\subsection{6/heartjnl-2019-BCS.10}

Introduction Echocardiography plays an essential role in the management of atrial fibrillation (AF) and the diagnosis of heart failure in these patients. Assessment of systolic and diastolic function is challenging in AF due to the irregular RR interval, resulting in variability from beat to beat. We compared the reproducibility of an index beat (figure 1) approach (similar RR intervals for the two prior beats before measurement) versus conventional averaging of three, five and ten consecutive beats in patients with permanent AF.

Methods Consecutive patients were evaluated with a Philips EPIC 7 and X5-1 transducer, and all measurements were analysed offline by an operator blinded to clinical details. A minimum of 30-beat loops were obtained of the apical 2, 3 and 4-chamber, lateral and septal e' and mitral E Vmax. Global longitudinal strain (GLS), Simpson's biplane ejection fraction (EF) and E/e' were calculated and the coefficient of variation determined between $3 \mathrm{x}$ index beats, averaged 3 beats, averaged 5 beats and averaged 10 beats. Intra- and inter-observer variability of GLS and E/e' were assessed in 30 and 10 patients respectively, using Bland and Altman analysis.

Results 50 patients with permanent AF and symptoms of heart failure were included, with $46 \%$ women and mean age $78 \pm 9$ years, heart rate $97 \pm 19 \mathrm{bpm}, \mathrm{NTpro}-\mathrm{BNP} 1173 \pm 647 \mathrm{ng} / \mathrm{L}$, NYHA class $2.5 \pm 0.6$ and BMI $30 \pm 6 \mathrm{~kg} / \mathrm{m}^{2}$. The index beat method had the lowest coefficient of variation (figure 1) for biplane ejection fraction, GLS and E/e' $(4.5 \%, 8.5 \%$ and $7.3 \%)$, when compared with the average of 3 beats $(11.6 \%$, $17.9 \%$ and $14.9 \%)$, average of 5 beats $(11.9 \%, 17.9 \%$ and $14.95)$ and average of 10 beats $(12.3 \%, 18.5 \%$ and $16.2 \%)$. GLS intra and inter-observer variability were lowest for the

\section{Index beat approach: RR intervals $\leq 60 \mathrm{~ms}$; cycle length $\geq 500 \mathrm{~ms}$}

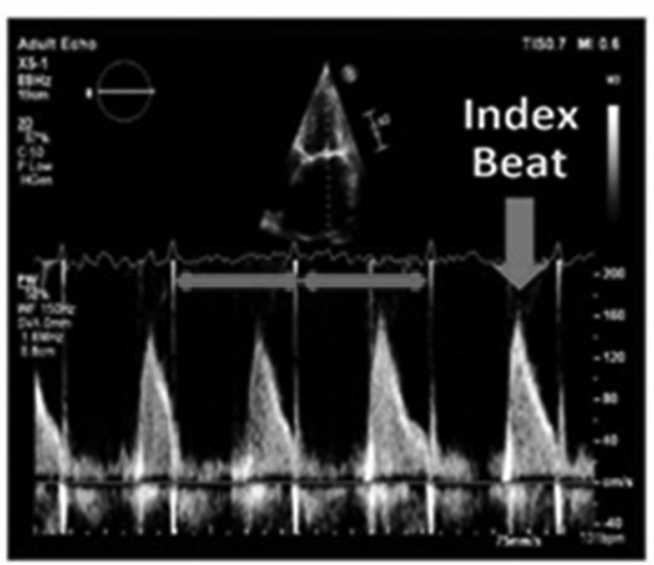

Index beat method compared to conventional averaging

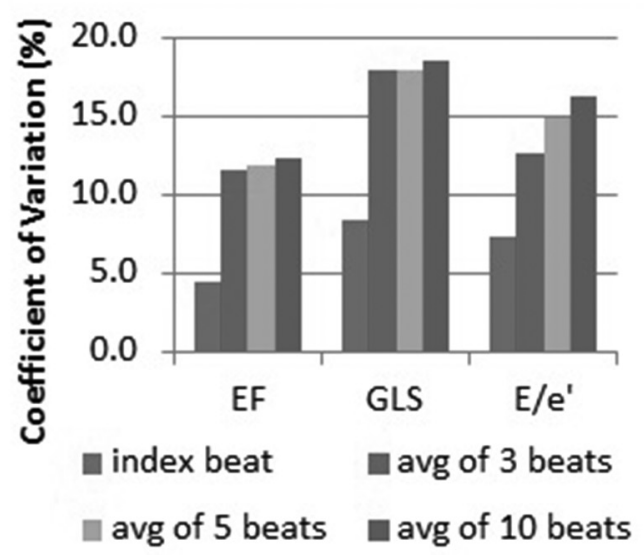

